\title{
Editorial - Volume 5, Number 3
}

Citation: Cannon, R. L. (2018). Editorial - Volume 5, Number 3. NeuroRegulation, 5(3), 84. http://dx.doi.org/10.15540/nr.5.3.84

Copyright: (c) 2018. Cannon. This is an Open Access article distributed under the terms of the Creative Commons Attribution License (CC-BY).
*Address correspondence to: Rex L. Cannon, PhD, BCN, Knoxville Neurofeedback Group, 7147 Kingston Pike, Ste 103, Knoxville, TN 37919, USA. Email: rcannonphd@gmail.com
Welcome to NeuroRegulation Volume 5, Issue 3. We appreciate your joining us for this issue.

NeurorRegulation and its editors are aware and concerned about the current issues facing neurofeedback, its organizations, and its practitioners. As with any organization, development and maturity come with much criticism and skepticism. The American Psychological Association (APA) underwent vast challenges, criticisms, and subsequent changes to become what it is today, yet there are persistent notions that "hard science" does not apply to psychological mechanisms. The International Society for Neurofeedback and Research (ISNR), the Association of Applied Psychophysiology and Biofeedback (AAPB), and the Biofeedback Certification International Alliance (BCIA) are organizations devoted to neurofeedback and its empirical status. It is very important that we focus on specific definitions, concepts, and standard metrics with well-defined parameters to further validate and replicate metrics used in current methods.

In the current issue Mark Jones and Heather Hitsman present data describing quantitative electroencephalography (qEEG)-guided neurofeedback for reducing symptoms of anxiety. Then, Ron Bonnstetter, Eric Gehrig, and Dustin Hebets present data examining gamma power asymmetry as an indication of response process validation. And finally, Ron Bonnstetter provides a book review of meditation and its effects on the brain and body.

NeuroRegulation appreciates these authors for their valuable contributions to the scientific literature for neurofeedback and learning. We strive for high quality and interesting empirical topics, which has led to this journal being indexed in Scopus, Elsevier Embase, and DOAJ. We encourage all those in the neurofeedback, biofeedback, and neuroscience disciplines to consider publishing with us. Our open-access format with no author processing fees and expedited publishing timelines (current average time from submission to publication is 50 days) makes NeuroRegulation a very favorable publication to submit your important work. We encourage researchers, clinicians and students to submit research findings, review articles, case reports, a new take on an important aspect of our field or a technical process, or even a review of a great book your colleagues should know about.

We thank you for reading NeuroRegulation!

Rex L. Cannon, PhD, BCN Editor-in-Chief

Email: rcannonphd@gmail.com

Published: September 29, 2018 